\title{
III.
}

\section{NEUE VERSUCHE}

mit dem Venturijchen hydraulifchen

$$
\text { Apparate, }
$$

von

\author{
J. A. E Y T E L W $W$ I N \\ Geheimen Oberbaurath in Berlin.
}

(Es ift aus den erften Gründen der Hydraulik bekannt, dals, wenn Wafter aus einem Gefalse durch ei-. ne kleine kreisförmige Oeffnuing, die fich in einer dünnen Platte betinder, ausftröurt, diefes, (wegen der Art, wie der Diuck fich in der daruiner ftehenden Walferläule for'planzt, a gelebn von fiörenden Einwilkungen,) uit der (refchwindigkeit gefchehen mülste, welche das ausfiefsende $W$ affutheilch, $n$ erlangen $w$ ürde, wenn es frei durch eine Höhe heralofele, die dem $W$ afferftande im Gefälse gleich ift. Setzt man die Wafferböhe über dem Mittelpunkte der Ueffnung h, die Gefc! windigkeit, womit das Waffer ausftrömt, $c$, die Fallbühe in der erften Sekunde $g$, und den Querfobnitt der Oeffnung $B$; Co müfote $c=+3$, oder $c=2 \sqrt{\mathrm{g} / 2}$ ley $n$, und, wofern das Waffer durch die kreisförmige Oeffnung in Feftalt eines $C y$ linders, der gleiche GrundGärbe mit der Oeffnung hat, ausftrömte. wärde in einer Sekunde eine $W$ affermenge $B .2 \sqrt{\xi} h$ ausfliefsen. Mir dieler hyputterijchen Gefchwindigkeit und Wollermenge firnmen indefs die wirklichen keinesweges überein; einmahl, weil der Wafferftrahl nicht als ein Cylinder, fondern conifch ausliefst und lich von der Mündung $a b$ beträchtlich verengert; und $z$ weitens, weil im Aus. 


\section{[ 296$]$}

Giefsen, víelleicht auch beim Zuftromen des Walters, noch kleine frörende Einwirkurgen ftatt linden. Der Durchmefler des fich verengernden Strabls an der Stelle der gröfsten Zufammenziehung ift, Vorfuchen zufolge, 0,8 vom Durchmel[er der Deffnung, folglich der Quer. Ichnitt deflelben $\frac{1}{2}{ }_{5}^{6}$ von der Plattenmündung, *) uad. es frörnt nur 0,619 von der hypothetifchen Waffermenge wirklich aus, fo dafs die Gelchwindigkeit in der Plattenöfinung felbft nur 0,619 . $c$ leyn kann.

Was in diefeon Auslielsen geändert wird, wenn man vor der Plattenöffnung Anfatzróhren verfchiedner Art anbringt, und was der Grund i it, warum cylindrifche und konilche Anfatzröhren den Ausfuls verfrärken; damit befchafrizte fich der erfte Theil ron $V$ entu $r i$ 's intereffanter hydrodynamifcher Abhanjlung, deren man fich aus Band II, Heft 4, und Band III, Heft $I$ ' und 2 diefer Anaalen erinnern wird. **) Venturi giebt dort. unter andern, Vorfchriften, wie man den Wafferausfluls gegen den durch eine Platte im Verbältnifle von 10: 24 vermeluren künne; Ceine Verfuche laffen aber gerade in diefem für dio Aowendung wichtigen Umftande vieles ziz wünfchea ïbrig. Sie lind es, welche H. Geheimer Oberbaur. Ey tel wein nach einer vervollkommneten Methode, mittelft eines ähnlichen Apparats als Ventu ri, nachdem er zuvor die Venturifchen Verluche felblt wiederhohlt und richtig béfunden hatte, durch die folgenden Verfuche auf das Genügendlte exgïnzt. Sie lind mir ron dem Herrn Verfalfer

-) Vergl. Annalen der Phyjlk, III, 38.

d. $H$.

- Unterfnchungen und Beobachtungen ïber die Seirenmitrheilung der Bewegang in Aülfigen Körpern, angewand c auf die Erklarung verfchiedner liydraulifcher ErCcheinanges, roa Venturi; in den Annaleri, II, 4:8 E. d. $H$. 


\section{$\left[\begin{array}{lll}297 & \end{array}\right]$}

eus feinem noch aicht erfchienenen Handbuche der $H_{\text {f. }}$. draulik gütiglt mitgetheilt worden, und werden dem Phyfiker um fo willkommner feyn, da der Druck diefes Hauptwerks in der Hydraulik to bald noch nicht vol. lendet (eya dürfte. *) Dieles als Einleitung.)

d. H.

$D_{\text {als fich die Waffermenge, welche aus einem Ge- }}$ fäfse durch eine kreisrunde Oeffnung ausftrömt, vermittelft angefetzter knnijcher Röhren; zelche fich gegen die Ausmündung verengern, in Vergleichung init andern Oeffuungen, anfehrilich vermehren läfst, zeigten fchon die Verfuche des Narchele J. Poleni, (de cajipllis, Flor. 17ıs.) Bei ejner Wallerhöhe von $1^{\prime} 9^{\prime \prime} 4^{\prime \prime \prime}$, und einer kreisrunden Oeffoung, filr welche die hypothetifche Walfermenge $27^{527}$ Kubikzoll betrug, wurden konifche $92^{\prime \prime}$ lange Kölren vor die Oeffinung geletzt, deren Ausmündung bei allen gleich, und zwar $2 f^{\prime \prime \prime}$ in Uurchmefler war. Als die finmindung nach einander $18^{\prime \prime \prime}, 60^{\prime \prime \prime}, 42^{\prime \prime \prime}, 5 j^{\prime \prime \prime}$ betrugs, war die wirklich ausfliefsende Walfermenge $0,5605,0,8844,0,8939$, 0,8992 ver hypothetilichen, in dafs hei ler konifchen Form im letzten veriuche cler Verluft des Walfers nur etwa $\frac{x}{s}$ ron cher hyputhetifchen Walfermenge war.

Gieht man der konilchen Anfatzröhre die Geftalt des zufammengezugenen Strahls bei Oefinungentn

-) Man verfl. die Anszügeaus Briefen am Ende diefes Hefss. d $H$.

Annal. d. Phyfik. B. 7. J. 18ar. Sr. j. 


\section{[ $\quad 298$ ]}

einer dannen Wand, fo dafs der Durchmeffer der Ausmindung $\frac{1}{5}$ vom Durchmeffer der Einınündung, und die Länge der Röhre etwas gröfser als der Halb. meller der Einmundung ift, (wie in Fig. 1 , Taf. V;) fo muls das Waffer eben fo ausfliefsen, wie durch den Quer $\{c h n i t t$ des zufammengezognen Strahls, vorausgefetzt, dafs die fcharfen Ecken der konifchen Rōhre etwras abgerundet find. Eine folche Röhre kann Mündung nach der Geftalt des zufammengezògenen Strahls, zur Abkürzung in der Folge, Mündung $\varphi$ heilsen.

Durch den kleinften Querfchnitt des zufammengezögnen Strahls lliefst ebeu lo viel Walfer, als durch die dazu gehörige Oeffnung in einer dünnen Wiand; daher mufs die Gelchwindigkeit in dem Querfchnitte in demfelben Verhältnilfe zunehmen, wie lein Flächeninhalt abnimmt. Nun ift der Querfchnitt des zufammengezognen Strabls $\frac{16}{25}$ voun Querfchnitte der Oeffnung; daher mufs die Gefchroindigkeit im Querfchnitte ier gröfsten Zulammenziehung, oder $c=\frac{1}{1} \cdot 0 ; 619 \cdot 2 \gamma^{\circ} g \sqrt{ } h=0,9672 \cdot 2 \sqrt{ }^{\circ} g V^{r} h$ feyn. Hat die Röhre $\varphi$ die erforderliche Geftalt, fo ift alfo auch die Gefchwindigkeit des Waffers in der Auslufsüffnung $E F$, (Fig. 1,) oder $c=$ $0,9672 \cdot 2 \sqrt{g} \sqrt{ } h=7,646 \sqrt{h}$. Fur den freien Fall eines Körpers wäre die Gelchıvindigkeit $=$ $2 \sqrt{ }:: g \sqrt{ } h$; hiernach verhält Gch die wirkliche $W a j=$ Jermenge, welche durch die Muindung $\varphi$ bei $E F$ ausäuft, zur hypothetifchen Waffermenge für die Oeffnung $E F$, wie $0,9672: 1$ oder nalie $=30: 31$, und 


\section{$\left[\begin{array}{lll}{[} & 299\end{array}\right]$}

es ift wahrfcheinlich, dafs beide Walfermengen gleich wären, wenn die Waflertheile nicht wegen der Adhälion an den Wänden der Röhre verzügert würclen, und wenn man $\Phi$ ganz genau die Geftalt des zufammengezogenen Strahls geben könnte.

Die Anfatzrühre $\varphi$ ift daher unter allen Ausflulsöffnungen von einer beftimmten Grölse clie vortheilhaftefte, weil das ausfliefsende Waller beim Ausgange eine folche Gefchwindigkeit in der Oeffnung $E F$ erlangt, welche nur wenig von derjenigen verfchieten ift, die ein Körper durch den freien Fall von cler Druckhöhe erreichen würde.

Nit einer folchen Mündung hat Venturi einen Verfuch angefteilt. $\left.{ }^{*}\right)$ Die Achfe feiner Röhre war horizontal, bei einer Druckihöhe von $52 \frac{1}{2}$ par. Zoll. Def Durchmeffer am Gefäfse hielt 18 , und bei der Ausmündung 14 Linien, die ganze Länge der Röhre 11 Linien, und man fand die Waffermenge fir eine Sekunde $=16465$ Kubikzoll. Die hypothetifche Waffermenge ift hier 176 Kubikzoll, daher die wirkliche 0,955 ron der bypothetilchen. Diefes nähert fich der rorbin gefumlnen Grenze $0,9^{b 7}$ fchon anfehnlich, und man wïrde fie erreicht haben, wean die konilche fiöhre nicht fcharfe Ecken gehabt bätte. ${ }^{* *}$ )

*) Verfuch 4; Annalen der Phyfik, 1I, 430. d. H.

**) Dies fcheint in allen Venturifchen Verfuchen der Fall gewefen zu feyn.

d. $H$. 


\section{[ 300 ]}

Aus meinen mit einer dergleichen Mündung ange(tellten Verfuchen, *) wenn die Einmündung 15 , die Ausmüntung 12, und die Länge der Röhre 8 Linien grofs war, finclet fich die wirkliche $W$ affermenge 0,9186 ron der hypothetíchen. Hierbei hatte die Münclung $\varphi$ ihre foharfen Ecken behalten. Nachdem aber diefe innerhalb fanft abgerundet ivaren, vermebrte fich die Waffermenge bis $0, y>9^{8}$ von der bypoihetichen, fo dafs fich nur ein geringer Unterfchied zwifchen beiden befand, und eine -grofsere Ausflufsmenge als durch die Venturifctien Verfuche bewirkt ward.'

Der Wafferverluft bei einerlei Ausmündung und gleicher Drucktıöhe ift hiernach

bei der Mündung $\varphi$ mit abgerund. Ecken $0,020 z ?$ v. der bei der Mündung $\varphi$ mit Tcharfen Ecken 0,0813 hypot. bei einer kurzen cylindrifch. Anfatzröhre 0,1875 S Wafbei einer Oeffnung in einer dünnen Wand 0,3810 ferm.

Es giebt noch ein Mittel, wodurch, obne Vermehrung der Druckhöhe, die Waffermenge, welclìe man durch eine beftimmte Ocffnung erhält, vermehrt werden kann. Statt der vorhin belchriebnen kunifchen Wüniungstücke, welche man kanifche Püt:ren der erfcen Art nennen kann, die foch gegen d:e Ausfursüffinung verengern, kann man folche koniche Röhren noch arifetzen, die fich nach dem Ausfiulje hin erweicern, fo dafs clie Einflufsöffnung $A B$,

*). Siehe weiter unten Erfahrung 2 and 3 ; und Ta. fel 1, No. 2, 3 .

E. 


\section{$[3 \circ \mathbf{I}]$}

(Fig. 2, Taf. V,) kleiner als tie Ausfluföffnung $E F$ itt, und die hier koniliche hubren der $z$ veiven Arc beifsen foilen.

Venturi hat hierüher wichtice Verfoche angeftellt. $\left.{ }^{*}\right)$ Die Einnändung $A B$ der, erweiterten konifchen Röhre $A B E F$ hatte bei allen leinen Verfuchen 15,5 Linien im Durchmelfer, fie befand fich aber nicht unmittelbar am Behälter, fondera zwifchen ihr und diefem war eine konifche höhre cer erften Art angebracht, welche beinahe die Gefialt des zufanmengezogenen Strahls hatte. Die Länge $A D$ und Ausmündung $E F$ wurden bei jedem Verfuche abgeändert, und man hatte bei unverän erter Druckhöbe von $32 \frac{\mathrm{T}}{2}$ Zoll die grölste Waffermenge, wenn $A D$ 148, $A B \quad 15,5$ und $E F 27 \mathrm{Li}$, ien grofs war. In diefem Falle erhielt man in jeder Sekinde 529,1 4 par. Kubikzoll, (Verf. 16, Annalen, II, 45o,) welches weit mehr ift, als die bypothetifche Waflermenge für eine Oeffnung vor $15 \frac{1}{2}$ Linien im parifer Maalse giebt. Diefe konifche Röhre der zweiten Art, in ihrer vortheilhafreften Geftult, mag hier, der Kürze baber, die Röhre $\psi$ heilsen.

Venturi befcireibt noch einen Verfuch, (Verf. 14. Annalen, II, 449,) bei welchem zwifchen der Miundung $\varphi$ und dieler konifchen Ausflufsröhre $\psi$, eine drei Zoll lange cylindirilche Röhre angebracht war, wodurch ebenfalls eine Vermehrung der Wal-

*) Satz V, Vexluch 13-17; Annalen der. Phyfhe, II, $448 \mathrm{f}$.

d. $H$. 


\section{$\left[\begin{array}{lll}302 & \end{array}\right.$}

fermenge bewirkt wurde. Weil aber keine Verfuche mit dieler konifchen Rühre der zweiten Art, $\psi$, ohne Verbindung mit andern Röhren befchrieben find, auch von der Vermehrung der Walfermenge bei einer drei Zoll langen cylindrifchen Röhre, durch Anfetzung der Rühren $\varphi$ und $\psi$, nicht geradezu auf längere Röhren gefchloffen werden kann, und daher die Behauptung Venturi's in Satz VII, (Annalen, II, 460,) daIs man bei einer cylindrifchen Röturenleitung, bei unveränderter Druckhöhe, durch zweckmälsige Anfatzröhren, ( $\varphi$ und $\psi$,$) allemahl$ die Waffernenge in. Verhältniffe $10: 24$ vermehren könne, fich uicht fo geradezu annehmen läfst; fo fchien es mir wichtig zu feyn, über diefe zur Erweiterung der Hydraulik und diefen für die Ausubung fo wichtigen Gegenftand, Verfuche mit der möglichften Genauigkeit anzuftellen.

$\mathrm{Zu}$ den folgenden Verfuchen diente mir ein 4 Fufs *) hoher prismatifcher Behälter, deffen horizontaler Durchfchnitt ein im Lichten 18,5 Zoll lasges und 14,4 Zoll breites Rechteck bildete. In der fchmalen vertikalen Seitenwand deffelben, befindet fich in einiger Entfernung vom Boden, eine meffingene Platte, welche mit der inoern Wand des Bebälters in einerlei Ebęne liegt, und in die man alle metallene Anfätze oder liöhren fo einfchrauben kann, dafs ihre Einmünclung in eben die Ebene fällt. Die

*) Alle hier gegebne Abmeffungén beziehen fich auf das rheinländifche Maals. 


\section{$\left[\begin{array}{ll}303 & ]\end{array}\right.$}

Einmündung konnte mittelft einer Klappe nach Gefalien geöfinet oder gefchloffen werder. Zur Beftinmung der Leit diente eine febr gut gearbeitete Sekunden-Pendeluhr, welche durch einen Zeiger die Sekunden bemerkte und mittellt einer Glucke durch Schläge hörbar marhte. ")

Sämmtliche Anjaczjücke und Röhren waren von Melfing gearbeitet, und die innere Fläche aufs genauefte polirt. Zur deichtern Vergleichurg der verfchiednen Refultate beziehen fich alle Oeffnungen a uf die Weite von einem Zolle, auch find alle Abineffungen mit dem hiefgen Originalmaalse verglichen. - Die cylindrifchen Röhren waren insgefammt einen Zoll weit; die Röhre $\bar{\varphi} 8$ Linien lang, und in der Einmündung 15, in der Ausmünclung 12 Linien oder 1 Zoll weit; und die Röhre $\psi$ $8 \frac{1}{1}$ Zoll lang, in der Einmündung 2 Zoll, und in der Ausmündung $1 \frac{19}{34}$ Zoll weit. Die Rühre $\bar{\varphi}$ in Verbiodung mit andern Röhren wurde nur bei der Einmündung, und $\psi$ bei der Ausmündung angebracbt.

Verlchiedene angeftellte Verfuche zeigten kleine Unregelmälsigkeiten, wenn man das Waffer im Bebälter, bei Beobachtung aller Vorficht, auf einerlei Höhe exhalten wollte, weil es fich fo leicht

*) Diele Uhr wurde vor dem Gebrauche nach dem Chronometer des Herrn Major von $\mathrm{Zac}$ h rectificirt, welcher fich damahls in der Verwahrung des Hra. Lieutenant von Textor befand. E. 


\section{[ 304 ]}

ereignet, dafs in gewilfen Augenblicken mehr oder weniger Walfer zugelalfen wird, als erforderlich ift. Auch war es unverneidlich, dafs nicht durch das zufliefsende Wafler eine unregelmälsige Beivegung in Bchälter entftand, weshalb ich es der Gellauigkeit, welche diefe Verfuche erfortern, angemeffener fand, heim Anfauge eines jeden Verfuchs eine Druckhöhe von 3 Fuls zu bewirken, und ohne Zuflufs den Walferfpiegel fo weit finken zu lalfen, bis ein Gefạ̈s von 4:56 Kubikzoll aogefüllt war. Hierdurch fenkte fich jedesmahl ider Waflerfpiegel im Bebälter, nach oft wiederhohlten Ausmeffungen, 15,6 Zoll, wodurch eben fo genaue Vergleichungen entltanden, als wenn die ,Druckhöhe unverändert geblieben wäre; auch hat man diefem Umftande die gute Uebereinftimmung der Verfuche mit einerlei Röhre zuzufchreiben.

Alle hier angeführten Verfuche find in Gegenvart des königl. Profelfors Herrn Hobert angeftellt oder wiederhoult worden.

Erfahrung 1. Kreisförmige 1 Zoll weite Oeffnung in einer $\Xi_{i}^{T}$ Zoll dicken Platte mit fcharten Kanten. Die beobachtete Zeit des Ausflulfes war in

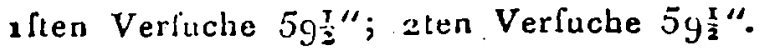

Erfahrung 2. Das Nundfück $\oplus$ beim Einflulle $1 \frac{1}{4}$ Zoll, beim Ausfluffe 1 Zoll weit, mit !charfen Kanten.

1. Verfuch $40^{\prime \prime}$; 2. Verfuch 40".

Erfahrung 3, Daffelbe Mundftück $\Phi$, wenn 


\section{$\left[\begin{array}{lll}305 & 1\end{array}\right.$}

die Ǩanten beim Ein - und Austulfe fanft abgerundet wareu.

1. Verfuch $\vec{\jmath}-\frac{T}{2} ;$ s. Verfuch

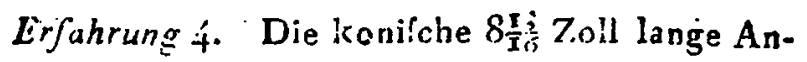
fatzröhre $\dot{\leftarrow}$, beim Einflulfe, ¿̈oll, beim Ausfluffe a? Zoll weit, mit feharfen Kunten.

1. Verluch $51^{\prime \prime}$ ?

1. Verfuch $\left.\left.31 \frac{1}{2}\right)^{\prime \prime}\right\} 3$, .

Erfahrung 5. Die Nundftücke $\varphi^{\prime}$ und $\psi$ ge• nau mit einander verbunden.
1. Veriuch 25 贸
2 . Verfuch $\left.2 q^{\prime \prime}\right\} 23 \frac{\pi}{3}$.

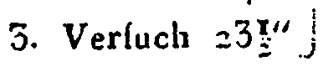

Erfahrung 6. Cylindrijche Röhre, 1 Zoll lang. Das Waffer folgte nicht den Wänden der Röhre.

$$
\text { 1. Verfuch } \bar{j} g g^{\prime \prime \prime} \text { : }
$$

Erfahrung 7. Cylindrifche Röhre, I Zoll lang, an der Einmündung mit $\bar{\psi}$ verbunden. Dus Waffer folgte den Wänden der Rülire.

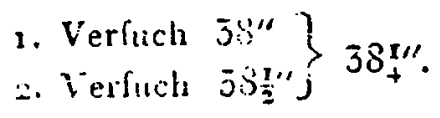

Erfahrung 8. Cylindrifche Rühre, I Zoll !ngg, bei der fiunündung init $\varphi$, bei der Ausmündung mit $\psi$ verbuiden.

$$
\text { 1. Verfucir } 2-T^{\prime \prime \prime} ;=\text { Verfuch } 2-\frac{I^{\prime \prime}}{2} \text {. }
$$

*) Wenn das Mundftúck oline weitere Bomerlsungen angeführt wird, fo ift jomer dasjanige wit 


\section{[ 306$]$}

Erfahrung 9. Cylindrifche Röhre, 3 Zoll lang. Das Walfer folgte nicht den Wänden der Röhre.

1. Verluch $59 \frac{\pi}{2}$.

Das Waffer folgte den Wänden der Rōhres

2. Verfuch $\left.45^{\prime \prime}\right\}$ 3. Verfuch $\left.444^{\prime \prime \prime}\right\} 44^{\prime \prime}$.

Diefelbe Röhre innerhalb des Bebälters angebracht, fo dafs fie von allen Seiten mit Waffer urngeben war, und ihre Ausmündung mit der innern Fläche des Behälters in einerlei Ebene lag.

4. Verfuch 45"; 5. Verfuch 45".

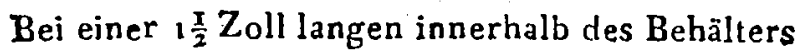
angebrachten Röhre, wobei das Wafler den Wän. den folgte, fand man diefelbe Zeit.

Erfahrung 10. Cylindrttche 3 Zoll lange Röh$\tau e$, mit der Einmündung $\varphi$.

1. Verfuch $\left.39^{\prime \prime}\right] 38_{4}^{3 \prime \prime}$.

Erfuhrung 11. Cylindrifche 3 Zoll lange Röhte, mit der Ausmundung $\psi$.

$\left.\begin{array}{l}\text { 1. Verluch } 33 \frac{1}{2} \prime \\ \text { 2. Verhich } 33^{\prime \prime} \\ \text { 3. Verfuch } 3 j^{\prime \prime}\end{array}\right\} 33 \frac{y^{\prime \prime}}{6}$.

Icharfen Kanten zu vaffehen, welches bei der Desten Erfubrung zu den Verfucbea diente. $E$. 


\section{[ 307 ]}

Erfahrung 12. Cylindrifche 3 Zoll lange Röbre, mit $\varphi$ und $\psi$.

1. Verfuch $27 \frac{5}{2} ;$ 2. Verfuch $27 \frac{70}{2}$.

Erfahrung 13. Cylindrifche 12 Zoll lange Röhre.

1. Verfuch $4 S^{\prime \prime} ; 2$. Verfuch $48^{\prime \prime}$.

Erfahrung 14. Cylindrifche 12 Zoll lange Röhre, mit $\varphi$.

1. Verfuch $42 \frac{\frac{T}{2}}{2} ; 2$. Verfuch $42 \frac{\frac{\pi}{2}}{2}$.

Erfahrung 15. Cylindrifche 12 Zoll lange Räb; re, mit $\psi$.
1. Verfuch $37 \frac{1}{2} "$,
2. Verfuch $\left.38^{\prime \prime}\right\} 377^{\prime \prime \prime}$.
3. Verfuch $57 \frac{11 \prime}{2} J$

Erfahrung 16. Cylindrifche 12 Zoll lange Röhre, mit $\varphi$ und $\psi$.

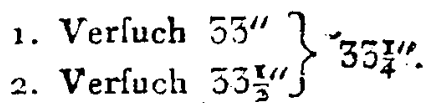

Erfahrang 17: Cylindrifche 24 Zoll lange Röhre.

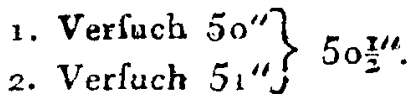

Frfahrung as. Cylindrifche 24 Zoll lange Röho re, mit $\varphi$.
1. Verfuch $46^{\prime \prime}$.

Erfahrung 19. Cylindrifche 24 Zoll lange Röb: re, mit $\psi$.
1. Verfuch, $40 \frac{\pi_{2}^{\prime \prime}}{2}$
2. Verfuch $\left.41^{\prime \prime}\right\} 40_{6}^{\prime \prime \prime}$
3. Verfuch $41^{\circ}$ J 


\section{$\left[\begin{array}{lll}308 & \end{array}\right]$}

Erfahrung 20. Cyliadrifche $2 千$ Zoll lange Röhre, mit $\varphi$ und $\psi$.

1. Verfuch $37 \frac{\mathrm{T}}{2} \div ; 2$. Verfuch $37 \frac{\mathrm{T}}{2}$.

Erfahrung 21. Cylindrifche 36 Zoll lange Röhre.

1. Verfuch $54^{\prime \prime} ;$ 2. Verfuch $54^{\prime \prime}$.

Erfahrung 22. Cylindrifche 36 Zoll lange Röbre, mit $\varphi$.

1. Verfuch $49 \frac{T}{3} " ; 2$. Verfuch $49^{\frac{\mathrm{T}}{2}}$.

Erfahrung 23. Cylindrilche 36 Zoll lange Rōhre, mit $\psi$. Das Wafler folgte nicht den Wänden der Röhre $\psi$, fondern nur dem Untertheile derfelbea.

$$
\text { 1. Verfuch } 52 \frac{I_{2}^{\prime \prime}}{2} \text {. }
$$

Wenn das Waffer genötbigt wurde, den Wänden der Rühre $\psi$ zu folgen.

2. Veifuch 44"; 3.Verfuch 44"; 4. Verfuch 44".

Erfuhruing 24. Cylindrifche 56 Zoll lange Röh$r e$, mit $\varphi$ unti $\psi$.

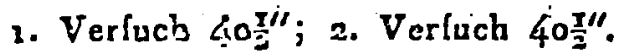

IIfahrung at. Cylisgatifcis- . Zoll lange Röhre.

1. Verfuch $58^{\prime \prime} ;$ 2. Verfuch $58 "$.

Erfahrung 26. Cylindrifcie 48 Zoll lange Röhre, nuit $\varphi$.

$$
\left.\begin{array}{l}
\text { x. Verfuch } 53 \frac{2}{2}{ }^{\prime \prime} \\
\text { 2. Verfuch } 53^{\prime \prime},
\end{array}\right\} 53_{4}^{\prime \prime \prime} \text {. }
$$




\section{[ 309$]$}

Erfalırung 27. Cylindrifche 48 Zoll lange Röhre, mit $\psi$. Das Waller folgte den Wänden der Rühre.

1. Verfuch $48^{\prime \prime} ; 2$. Verfuch $48^{\prime \prime}$.

Erfahrung 2S. Cylindrifche fo Zoll lange Rölure.

3. Verfuch 61 "; 2. Verfuch 61 ".

Erfahrung 29. Cylindrifclie 6o Zoll lange Röhre, mit $\bar{\varphi}$.

$$
\left.\begin{array}{l}
\text { 1. Verfuch } 57^{\prime \prime} \\
\text { 2. Verfuch } \left.56 \frac{1}{2}\right]
\end{array}\right\} 6_{+}^{2 \prime \prime}
$$

Erfahrung כo." Cylindrifche Go Zoll lange Pühre, mit $\psi$. Das Walfer folgte den Wänden der Rühre $\psi$, aufser etwa $\frac{1}{3}$ des Obertheils blieb unausgefüllt, und das Waffer war durch keinen Kunftgriff lahin zu bringen, dafs es die Röhre ganz ausfüllte.

1. Verfuch $52^{\prime \prime} ; 2$. Verfuch $52^{\prime \prime}$.

Um die vorftehenden Erfabrungen beffer $2 . u$ überfehen und auf eine gemeinfchaftliche Einheit zuriickzuführen, darf man nur die Zeit beftimmen, in welcher bei der anfânglichen Druckhöhe von $3 \mathrm{Fufs}$ und den übrigen bekannten Abmeffungen, 4156 Kubikzoll Waffer durch eine I Zoll weite krei:für. mige Oefinung ablaufen, indem man vorausfetzt, dals weder Contruction noch andere Hinderniffe die Bewegung des Walfers aufhalten, fondern daffelbe eben die Gefchwindigkeit in der Oeffnung, wie ein frei fallender Körper erlangt. Dieles giebt die Zeit. 


\section{[ 310 ]}

für die hypothetifche Waffermenge $=36,77^{\circ}$. Da nun die Zeiten des Ausfluffes gleicher Waffermengen, bei gleichen Gefälsen ohne Zuflufs, die fich mit verfchiedner Contraction ausleeren, umgekehrt wie die Waffermengen verhalten, welche bei unverằnderten Druckhöhen und mit derfelben Contraction in gleichen Zeiten auslaufen würden; ${ }^{*}$ ) fo giebt diefes ein leichtes Mittel, bei fämmtlichen vorftehenden Erfahrungen anzugeben, wie fich die Wajfermenge, welche bei unveränderter Druckhöhe ausgelaufen züre, zur hypotketifchen verhält.

*) Wenn $T$ die Zeit ift, in welcher fich das Gefäls, deffen Querfchnitt $A$ und Auslufsöffnung $a$ ift, ohne Contraction bei der anfanglichen Druckhöbe $h$ um die Tiefe $k$ ausleert, und $t$ diefe Zeit für eine beftimmte Contraction bei eben diefem Gefilise bezeichnet; und wean ferner bei unveränderter Druckhöhe $h$ in der Zeit $\boldsymbol{r}$ ohne Contraction die Waffermenge $M$, und in eben der Zeit mit Contraction die: Waffermenge $m$ auslauft; und wenn endlich $\propto$ den Coefficienten bedeutet, womit $\sqrt{ } h$ multiplicirt werden muls, damit man $c$ erhalte : lo ift nach hydraulifcben Gründen

$$
\begin{aligned}
& T=\frac{2}{2 \sqrt{g}}[\sqrt{ } k-r(h-k)] \frac{A}{a} \text { und } \\
& t=\frac{2}{\alpha}_{\alpha}[\sqrt{ } h-r(h-k)] \frac{A}{a} .
\end{aligned}
$$

Ferner $M=t a 2 \sqrt{g} \sqrt{ } h$ und

$$
\begin{aligned}
& m=\tau a \propto \sqrt{ } h ; \text { daber verhält fich } \\
& T: t=\propto: 2 \sqrt{g} \text { und } \\
& m: M=\alpha: 2 \sqrt{ }: \text {; folglich } \\
& T: t=m: M_{1},
\end{aligned}
$$




\section{[ $3 \mathbf{I t}$ ]}

In der folgenden erfien $T a f e l$ bedeutet $\varphi$ die Einmündung mit fcharfen, $\Phi^{\circ}$ diefelbe mit abgerundeten Kanten, und $\psi$ die Ausmündung. In den Verfurben, wobei diefe Buchftaben ftehn, wurden diele Anfatzröhren gebraucht, in den übrigen nicht. Das hinterfte Ende der Einmündungen, das vorderfte der Ausmündungen, und alle cylindrifche Röhren waren genau 1 Zoll weit; die Zahl von Zollen in der zweiten Spalte zeigt die Länge der angewandten cylindrifchen Röhre an. Die wirkliche Waflermenge in der letzten Spalte ift in Theilen der hypothetifchen Waflermenge, als Einheit, ausgeetrückt. 


\section{[ 3I2]}

Erfte Tafel.

\begin{tabular}{|c|c|c|c|}
\hline $\begin{array}{l}\text { Erfah. } \\
\text { rung. }\end{array}$ & $\begin{array}{c}\begin{array}{c}\text { Anfatzröh- } \\
\text { ren. }\end{array} \\
\end{array}$ & $\begin{array}{c}\text { Benbachtete } \\
\text { Zeit des } \\
\text { Anstiffes, } \\
\text { Sekunden. } \\
\end{array}$ & $\begin{array}{c}\text { Wirkliche } \\
\text { Wiffermenge } \\
\text { in Theilen } \\
\text { der hypothe- } \\
\text { tilchen.. }\end{array}$ \\
\hline 1 & $\left(\frac{1}{4}\right)^{\prime \prime} ;$ & $90: "$ & 0,6176 \\
\hline 2 & $90^{\prime \prime}$ & $40^{\prime \prime}$ & 0,9186 \\
\hline 3 & $\varphi^{\prime} \circ$ & $37 \frac{1}{2}$ & 0,9798 \\
\hline 4 & $\circ \psi$ & $31 \frac{1}{7}$ & 1,1758 \\
\hline 5 & 904 & $23 \frac{2}{7}$ & 1,556 \\
\hline 6 & $1^{\prime \prime}$ & $59 \frac{1}{3}$ & 0,6176 \\
\hline 7 & $\Phi \quad I$ & $38 \div$ & 0,9606 \\
\hline 8 & $\begin{array}{lll}\varphi & 1 & \psi\end{array}$ & $27 \frac{1}{2}$ & 1,3362 \\
\hline 9 & $3^{\prime \prime}$ & $447^{11}$ & 0,8211 \\
\hline 10 & $\Phi 3$ & 384 & 0,9482 \\
\hline II & $3 \psi$ & $33 \frac{1}{6}$ & 1,1079 \\
\hline 12 & $\$ 3 \downarrow$ & $27 \frac{1}{2}$ & 1,3362 \\
\hline 13 & $12^{\prime \prime}$ & $48^{\prime \prime}$ & 0,7055 \\
\hline 14 & 912 & $42 !$ & $0,864^{6}$ \\
\hline Is & $12 \psi$ & $37 \frac{\frac{1}{2}}{2}$ & 0,9798 \\
\hline 16 & $\$ 12 \downarrow$ & $33 \frac{\pi}{4}$ & 1,1051 \\
\hline 17 & $24^{\prime \prime}$ & $50 ! 3$ & 0,7276 \\
\hline 28 & $Q 2+$ & 46 & 0,7 y8 \\
\hline I9 & $24 \psi$ & $40 \frac{5}{\pi}$ & 0,8999 \\
\hline 20 & $924 \psi$ & $37 \frac{\pi}{2}$ & $0,6.798$ \\
\hline 21 & $30^{\prime \prime}$ & $54^{\prime \prime}$ & $0,6 \times 04$ \\
\hline 22 & 936 & $49 \frac{1}{2}$ & 0,7523 \\
\hline 23 & $36 \downarrow$ & 44 & 0,8351 \\
\hline 24 & 9364 & $40 \frac{1}{2}$ & 0,9073 \\
\hline 25 & $43^{\prime \prime}$ & $58 \%$ & 0,6335 \\
\hline 26 & 948 & 537 & 0,6900 \\
\hline 27 & $48 \downarrow$ & 48 & $0,76,5$ \\
\hline 28 & $60^{\prime \prime}$ & $61^{11}$ & 0,3024 \\
\hline 29 & 960 & $56 \frac{7}{4}$ & $c, 6+75$ \\
\hline 30 & $60 \downarrow$ & 52 & 0,7066 \\
\hline
\end{tabular}

In de: vorftehenden Tafel fond lämnitliche Verluche nach der Länge der einen Zoll weiten Rühren geordnet. Stellt man aber diejenigen Verfuche zulammen, welche Gch auf Rübren von einerlei Art 


\section{$\left[\begin{array}{lll}3 & 13\end{array}\right]$}

beziehen, fo entftehen zur beffern Vergleichung noch folgende vier Tafeln.

\section{Zweite Tafel.}

Cylindrifche Röhren ohne Einmündung oder Ausmündung.

\begin{tabular}{|c|c|c|c|}
\hline & $\begin{array}{l}\text { Länge der } \\
\text { cylindri- } \\
\text { fchen Röhre, } \\
\text { Zoll. }\end{array}$ & $\begin{array}{l}\text { Beobachtete } \\
\text { Zeir des Aus } \\
\text { Gunfes, } \\
\text { Sekunden. }\end{array}$ & $\begin{array}{l}\text { Wirklicbe } \\
\text { Waffermenge } \\
\text { in rheileg } \\
\text { der hypothe- } \\
\text { tifchen. }\end{array}$ \\
\hline I & $\left(\frac{1}{4}\right)$ & $59 \frac{\pi}{2}$ & 0,6176 \\
\hline & I & $59 \frac{4}{2}$ & $0,6 \times 76$ \\
\hline & 3 & $44 \frac{3}{7}$ & $0,82 \pi x$ \\
\hline & 12 & 48 & 0,7655 \\
\hline & 24 & $50 \frac{x}{2}$ & 0,7276 \\
\hline & 36 & 54 & 0,8804 \\
\hline & 48 & 58 & 0,6335 \\
\hline & & & 0,6024 \\
\hline
\end{tabular}

\section{Dritte Tafel.}

Cylindrifche Röhren mit der Einmändung $\varphi$.

\begin{tabular}{|c|c|c|c|}
\hline & $\begin{array}{c}\text { Länge der } \\
\text { cylindri } \\
\text { fchen Rlohr } \\
\text { Zollh. }\end{array}$ & $\begin{array}{l}\text { Beobachrete } \\
\text { Zeic des Atus } \\
\text { furfes, } \\
\text { Sekunden. }\end{array}$ & $\begin{array}{l}\text { Wirk liche } \\
\text { Warfermenge } \\
\text { in rheilen } \\
\text { der hypothe } \\
\text { tifchen. }\end{array}$ \\
\hline & & & 0,9196 \\
\hline & & & \\
\hline & & & 12 \\
\hline & $\begin{array}{ll}9 & 12 \\
0 & 24\end{array}$ & & \\
\hline & $43^{6}$ & 48 & 0 \\
\hline & $\begin{array}{ll}9 & 48 \\
9 & 60\end{array}$ & $\begin{array}{c}53 \frac{1}{13} \\
56 \frac{3}{4}\end{array}$ & 0,6475 \\
\hline
\end{tabular}

Annal. d. Phyfik, Bo7. J. 1801. St. 3. 


\section{[ 314 J}

\section{Vierte Tafel.}

Cylindrifche Röhrèn mit der Ausmün. dung $\dot{\psi}$.

\begin{tabular}{|c|c|c|c|}
\hline & $\begin{array}{l}\text { Läge der } \\
\text { rylinidri- } \\
\text { rchen Riinre. } \\
\text { Zull. }\end{array}$ & $\begin{array}{l}\text { Banbachterte } \\
\text { Zeit Nes Alis } \\
\text { H.rfes. } \\
\text { S. kunden. }\end{array}$ & $\begin{array}{l}\text { Wirkliche } \\
\text { IVaffermenge } \\
\text { in Thrilen } \\
\text { der hypothe- } \\
\text { cifchen. }\end{array}$ \\
\hline 1 & 04 & $31 \frac{1}{4}$ & 1,1759 \\
\hline 2 & 34 & $33 \frac{1}{6}$ & 1,1079 \\
\hline 3 & 224 & $37 \frac{\pi}{3}$ & $0,9 \cdot 98$ \\
\hline & $24 \dot{\psi}$ & $40 \frac{\pi}{6}$ & 0,8909 \\
\hline 5 & $36 \dot{\psi}$ & 44 & 0,8351 \\
\hline & & 48 & 0,7635 \\
\hline 7 & 6 & 52 & 0,7066 \\
\hline
\end{tabular}

Finfte Tafel.

Cylindrifche Köhren mit der Einmündung $\bar{\varphi}$ und der Ausmündung. $\psi$.

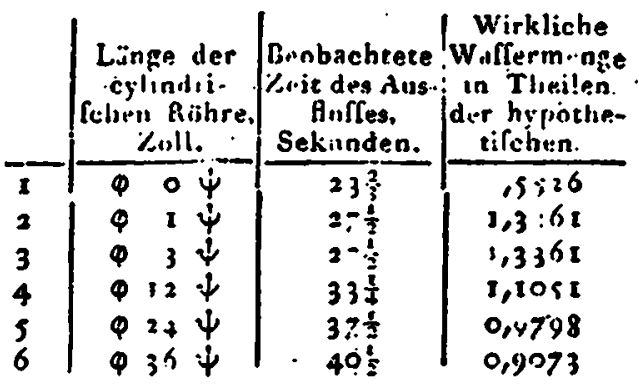

Die in diefen Tafeln georilneten Erfạhrungen berechrigen uns r.u f:.l enden Schliulfen.

1. rituter illivi:ens gleichen Uinftänden verialten fich d:e Waffermengen, die aus einer Oeffnurig in einer ditruen llant, zu denen, die ans vier Mindung $\psi \vec{\psi}$ : nich cler form des zulammengezoge" nen Strails, ausliefsen, wenn die Aasmündung 


\section{[ $3 \times 5]$}

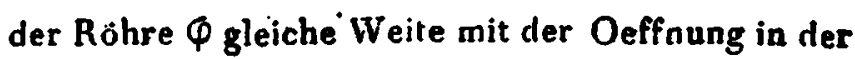
dinnen Wand hat, wie $40: 59 \frac{\pi}{2}$, das ift, wie $1: 1, \dot{4} 87$;

und find die feharfen Kanten der Mündung $\varphi$ abgerundet, wie $57 \frac{1}{2}: 59 \frac{1}{2}$, oder wie. $1: 1,587$.

2. Bei einer $O$ rffunng in einer dünnen Wand, zur Nia:durg $\downarrow$, wenn die Einmünilung der Rühre $\downarrow$ der Oeffrung in der dlinnen Wand gleich ift, wie $31+5$ : 39 , oller wie $1: 1,004$.

3. Rei einer Oeffnutrg in einer dünnen $W$ and, zu der aus den Röhren $\varphi$ und $\psi$ zufammengefetzten Mitnlung, wie $25 \frac{3}{3}: 59 \frac{x}{2}$, dds ilt, wie $1: 2,514$.

Es ift bemerkenswerth, dafs durch diefe $Z u$ -

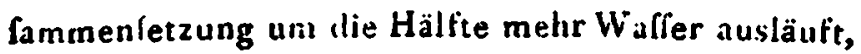
als wenn das Waller wie ein frei fallender Korper befchleunigt würcle.

4. Die Waffermenge bei einer kurzen cylindriichen Anfatzrötare, verbält fich zu der, mit der kurzen Anfatzröbre verbundnen Finmindung $\varphi$, wie 38: : 4千, oder wie $1: 1,1.54$.

5. Bei ejner kurzen cyliudrifchen Anfazzröhre, zu dieler Röhre mit der Ausmïndung $\psi$ rerbunden, wie $35^{5}, 4^{3}$, oder wie $1: 1, \bar{j}+9$.

6. Bej einer kurzen Anfatzröhre, zu dieler mit der E.in- und Ausmindung $\phi$ und $\psi$ verbundenen Rijhre, wie $27 £:\left\{4 \frac{1}{4}\right.$, das ift, wie $1: 1,6 \% 27$.

So weit diefe Schlülfe von Oetfinungen in einer dünnen Wand oder ron kurzen Anlatzröhren gelten, künnen lie durch die befchriebnen Verfuche gerechtfertigtwerden; wenn aber Venturi in feinen Un- 


\section{[ 316$]$}

terfuchungen etc., Satz VII, (Annalen der Phyfik, II, 46o, behauptet, dafs man durch angemeffene Einund Ausmùndungen bei jeder cylindrifchen Röhre die. Waffermenge im Vertältnille von 10 zu 24 rermehreo könne, und fich dieferhalb: auf feine Verfuche mit 3 Zoll langen Röhrea beruft; fo ift offenbar der Schlufs von kurzen Anfatzröhren zu weit ausgedehnt, wenn er von jeder cylindrifchen Rühre gelten foll.

Dafs bei längern Röhren die Wallermenge nicht in einem eben fo grofsen Verhältnilfe vermehrt wiril, wie bei kurzen Anfatzröhren, beweilen meine Verfuche hinjänglich, und es mufs irgend eine Rïhrenlänge geben, wo die Mündungen $\varphi$ und $\psi$ gar keine Vermehrung der $W_{a}$ ffermenge bewirken.

Vergleicht man die Waffermengen der zweiten Tafel mit denen der dritten, fo ftehen die Vermehrungen, welche durch die Einmündung $\grave{\varphi}$ bewirkt werden, in folgenden Verbältniflen:

Länge der Röhre:

$$
\begin{aligned}
& 3 \text { Zoll } 38 \frac{3}{3}: 44 \frac{3}{5}=1: 1,154 \\
& 12-42 \frac{3}{2}: 48=1: 1,129 \\
& 24-46: 50 \frac{5}{2}=1: 1,098 \\
& 36-44 \frac{7}{2}: 54=1: 1,092 \\
& 48-53 \frac{3}{4}: 58=1: 1,089 \\
& 60-56 \frac{7}{4}: 61=1: 1,075
\end{aligned}
$$

woraus hervorgeht, dafs die Münilurg $\varphi$ die Waffermenge bei larigen Riöhren nicht eben fo vermehrt, wie beı kurzen Anlatzrühren. 


\section{$\left[\begin{array}{lll}17 & \end{array}\right]$}

Daffelbe gilt von der Ausmündung $\psi$.

Länge der Röhre:

$$
\begin{aligned}
& 3 \text { Zoll } 33 \frac{x}{6}: 44 \frac{1}{4}=1: 1,379 \\
& 12-37 \frac{1}{2}: 48=1: 1,180 \\
& 24-40 \frac{5}{6}: 50 \frac{x}{2}=1: 1,236 \\
& 36-44: 57=1: 1,217 \\
& 48-48: 58=1: 1,208 \\
& 60-52: 61=1: 1,173
\end{aligned}
$$

Aehriliche Abnahme in der Vermehrung der Waffermenge findet mau für längere Röhreo, wenn die Nünilungen $\Phi$ und $\psi$ zulammen angebracht werden. Auch habe ich zur Ueherzeugung, dals hei einer gewiffen Länge der Röhre die Mündung $\psi$ keine Vermehrung der Waffermenge bewirke, unter 3 Fufs Druckhöhe, mit einer 20 Fuls langen Ruhre Verluche angeftellt, bei welcher immer eben diefelbe Wallermenge in gleicher Zeit erbalten wurde, man mochte $\psi$ anbringen oder nicht; auch war es nicht möglich, zu bewerkftelligen, dals das Waller die ganze Röhre $\psi$ ausfullte, weil es fich immer von dem obern Theilc derlelben losrifs.

Wenn es nun gleich wabricheinlich ift, dafs fir kleinere Gefchwindigkeiten des ausfliefsenden Walfors die Weite der Ausmündung der Rühre $\psi$ kleiner werden mufs, fo läfst fich doch abfehen, dafs, wenn hierdurch auch eine geringe Vermehrung ier Waffermenge bewirkt wird, diefe docb nie fo beträchtlich feyn kann, wie fie Venturi angiebt. 